\title{
CUBES WITH KNOTTED HOLES
}

\author{
BY \\ R. H. BING AND J. M. MARTIN( $\left.{ }^{(}\right)$
}

\begin{abstract}
The statement that a knot $K$ has Property $\mathrm{P}$ means that (1) if $C$ is a cube with a $K$-knotted hole, and $M^{3}$ is a simply connected 3-manifold obtained by sewing a solid torus onto $C$, then $M^{3} \cong S^{3}$, and (2) any piecewise linear homeomorphism of a cube with a $K$-knotted hole into $S^{3}$ can be extended to a piecewise linear homeomorphism of $S^{3}$ onto $S^{3}$. In this paper it is shown that many familiar classes of knots have Property P.
\end{abstract}

1. Introduction. The 3-dimensional Poincare conjecture is that a compact, connected, simply connected 3-manifold without boundary is topologically a 3sphere $S^{3}$. Despite efforts to prove the conjecture, it has withstood attack [4].

Since no solution has been found, some believe that the conjecture is false, and that there is a counterexample.

In attempting to construct a counterexample to the Poincare conjecture, the first hurdle seems to be that of showing that the proposed example has a trivial fundamental group. This is difficult in that the information about the fundamental group usually appears as an exotic presentation of the group. Having somehow verified that this group is indeed trivial, one encounters the second hurdle of somehow showing that the example is topologically different from $S^{3}$.

It is known that every orientable 3-manifold may be obtained by removing a collection of disjoint solid tori from $S^{3}$ and sewing them back differently [14], [22], [12]. In this paper we examine some of the possibilities for constructing a counterexample to the Poincare conjecture by removing a single solid torus from $S^{3}$ and sewing it back differently. Actually, we examine not only this process but one analogous to it which we call "attaching a pillbox to a cube with a knotted hole".

We find it useful to consider two sorts of surgery which are inverses of each other. If $M^{3}$ is a 3-manifold with boundary, $A^{2}$ is an annulus on $\mathrm{Bd} M^{3}$ and $P^{3}$ is a 3-cell such that $M^{3} \cap P^{3}=A^{2}=\mathrm{Bd} P^{3} \cap \mathrm{Bd} M^{3}$, then we say that $M^{3} \cup P^{3}$ was obtained by sewing a pillbox $P^{3}$ to $M^{3}$ along $A^{2}$. Suppose that $H^{3}$ is a 3-cell in $M^{3}$ such that $H^{3} \cap \mathrm{Bd} M^{3}$ is the union of two disjoint disks $D_{1}^{2}$ and $D_{2}^{2}$, and $N^{3}=\mathrm{Cl}\left(M^{3}-H^{3}\right)$ is a 3-manifold with boundary. We say that $N^{3}$ was obtained by boring $a$ hole in $M^{3}$.

Received by the editors September 29, 1969.

AMS 1970 subject classifications. Primary 55A05, 55A25, 55A40.

Key words and phrases. Knots, cubes with knotted holes, Poincaré conjecture, 3-manifolds, Property $\mathbf{P}$.

(1) The first author was partially supported by ONR grant NR $043-374$, and the second by NSF grant GP-3857. 
If $M^{3}$ is a 3-cell, the manifold $N^{3}$ described in the preceding paragraph is called a cube with a hole. We name the hole after a polygonal simple closed curve $h(J)$ in Euclidean 3-space, $E^{3}$, where $h$ is a homeomorphism of $M^{3}$ into $E^{3}$, and $J$ is a simple closed curve on Bd $N^{3}$ which is the union of an arc from $D_{1}^{2}$ to $D_{2}^{2}$ on $\mathrm{Bd} H^{3}$ and an arc from $D_{1}^{2}$ to $D_{2}^{2}$ on $\mathrm{Bd} M^{3}$. Thus, we speak of a cube with a trefoil (overhand) knotted hole, a cube with a figure eight knotted hole, etc. If $K$ is a knot, then a cube with this type of hole is called a cube with a K-knotted hole and is denoted by $C^{3}(K)$. A cube with an unknotted hole is topologically a solid torus, that is, the Cartesian product of a disk and a circle. We reserve the terminology "cube with a knotted hole", for a manifold of this type different from a solid torus. One obtains the fundamental group of $N^{3}$ by computing the fundamental group of $E^{3}-h(J)$ since $\pi_{1}\left(N^{3}\right)=\pi_{1}\left(E^{3}-h(J)\right)$.

Let $M^{3}$ be a compact, connected 3-manifold with boundary whose boundary is a 2-sphere. Then $M^{3}$ is a homology 3-cell if its 1st homology group is trivial and a homotopy 3-cell if its fundamental group is trivial. The Poincaré conjecture is equivalent to the conjecture that each homotopy 3-cell is homeomorphic with a 3-cell.

Poincaré's original conjecture was [19] that the homology of a compact combinatorial $n$-manifold determines its topology. This would have implied that each homology 3-cell is a 3-cell, but Poincaré himself obtained [20] a counterexample to this shortly after he made the original conjecture. Peculiar homology 3-cells can be built by sewing pillboxes onto cubes with knotted holes so that the connecting annuli run through the holes.

One way to try for a counterexample to the Poincaré conjecture is to seek a homotopy 3-cell obtained by sewing a pillbox onto a cube with a knotted hole so that the connecting annulus runs through the hole in the cube (perhaps several times). It is known that no homotopy 3-cell results by using a trefoil knot [2] or even a torus knot [11], [13]. We show in this paper that none results for many other sorts of knots. We do not know the answer for all knots despite an assertion made on p. 36 of [2]. See [3].

The preceding paragraph implies that if $K$ is a torus knot and $C^{3}(K)$ is a polyhedron in $E^{3}$ which is a cube with a $K$ knotted hole, then each piecewise linear homeomorphism of $C^{3}(K)$ into $E^{3}$ can be extended to a piecewise linear homeomorphism of $E^{3}$ onto itself. We show in this paper that many other knots have this property. A counterexample to the Poincaré conjecture would be at hand if one could find a knot $J$ with this property and such that $C^{3}(J)$ could be changed to a homotopy cube by sewing on a pillbox along an annulus that runs through the hole.

We say that a knot $K$ has Property $\mathrm{P}$ if (1) each homotopy 3-cell obtained by sewing a pillbox onto $C^{3}(K)$ is a 3-cell, and (2) if $C^{3}(K)$ is a polyhedral cube with a $K$-knotted hole in $E^{3}$, then any homeomorphism of $C^{3}(K)$ into $E^{3}$ can be extended to a piecewise linear homeomorphism of $E^{3}$ onto itself. The trivial knot satisfies the first restriction but not the second. In $\$ \S 2-5$, we extend the class of knots known to 
have Property P. We are advised by Ralph Fox that some of the results in this paper have been obtained independently by F. Gonzales in his Princeton thesis.

2. Cube with a figure eight knotted hole. The figure eight knot, $T_{2}$, shown in Figure 1 is a special kind of a twist knot, $T_{n}$ to be considered later. However, the study of $C^{3}\left(T_{2}\right)$ has an interesting history, and so is treated independently.

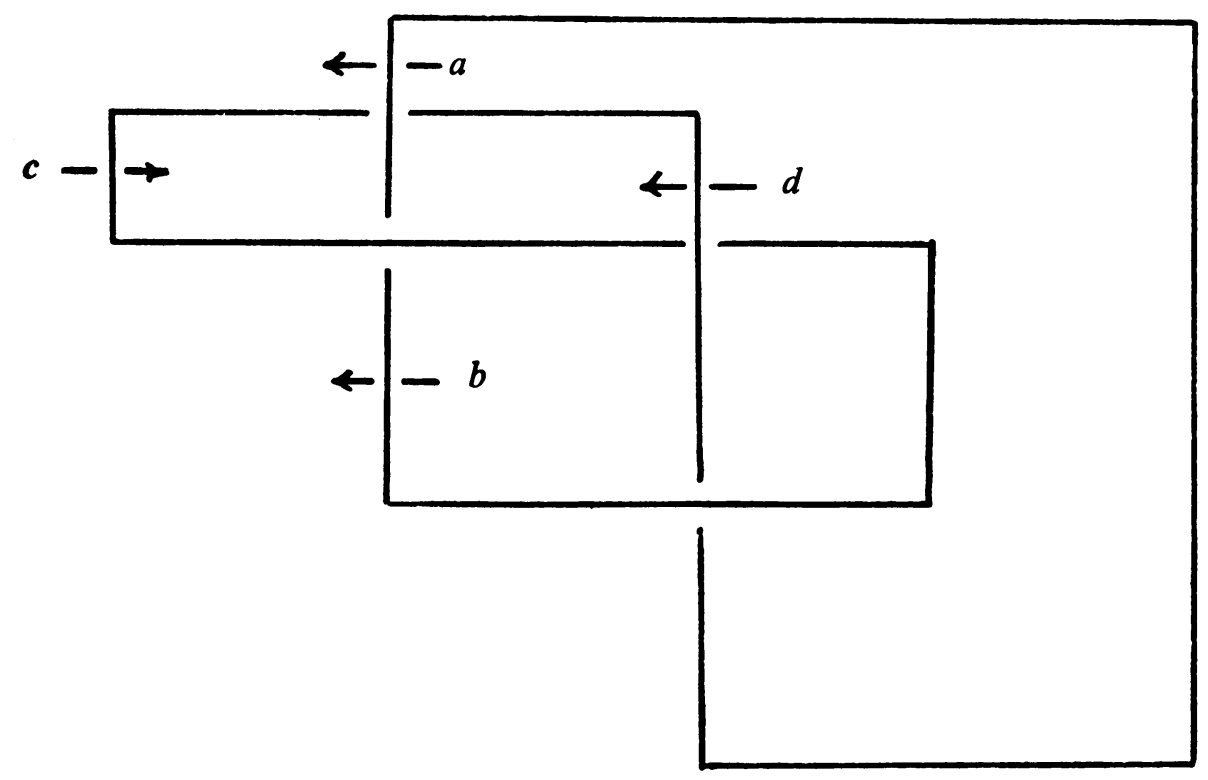

FIGURE 1

If one reads the fundamental group of $E^{3}-T_{2}$ from Figure 1 as suggested in [5] or [10], it is found to have a presentation

$$
\pi_{1}\left(E^{3}-T_{2}\right)=\left\{a, b, c, d ; a c a^{-1} d^{-1}=a c^{-1} b^{-1} c=d c^{-1} d^{-1} b=d b a^{-1} b^{-1}=1\right\} .
$$

Since $d=a c a^{-1}$ and $b=c a c^{-1}$, the presentation simplifies to

$$
\pi_{1}\left(E^{3}-T_{2}\right)=\left\{a, c: a c a^{-1} c^{-1} a c^{-1} a^{-1} c a c^{-1}=1\right\} .
$$

This is also the fundamental group of $C^{3}\left(T_{2}\right)$.

If one forms a 3-manifold $C^{3}\left(T_{2}\right) \cup P^{3}$ by sewing a pillbox $P^{3}$ to $C^{3}\left(T_{2}\right)$ along an annulus that runs through the hole $n$-times, one finds that the relation $\left(b^{-1} a d^{-1} c\right)^{n} a^{i}=1$ is added. The abelianization of the resulting group yields the integers mod $|i|$, so we find that in order to construct a homology cube we must have $i= \pm 1$. Hence

$$
\begin{aligned}
\pi_{1}\left(C^{3}\left(T_{2}\right) \cup P^{3}\right) & =\left\{a, c ; a c a^{-1} c^{-1} a c^{-1} a^{-1} c a c^{-1}\right. \\
& \left.=\left(c a^{-1} c^{-1} a^{2} c^{-1} a^{-1} c\right)^{n} a^{ \pm 1}=1\right\}
\end{aligned}
$$

Letting $n$ take on negative values as well as positive ones, we drop the \pm sign from the final exponent on $a$. 
The question was raised in [3] as to whether or not, for some $n \neq 0, C^{3}\left(T_{2}\right) \cup P^{3}$ might be a homotopy 3-cell. In the hopes of getting something started, Bing raised the question in [4] as to whether or not it might be a homotopy 3-cell in the special case where $n=-1$. Independent answers were received in letters to Bing for the question about the special case. In $1964 \mathrm{~J}$. Mennicke wrote Bing that in the special case no homotopy 3-cell results since for $n=-1$ there is a homomorphism of $\pi_{1}\left(C^{3}\left(T_{2}\right) \cup P^{3}\right)$ onto the simple group of order 168. Later J. M. Martin reported a similar result. In 1967 W. R. Alford and D. E. Penny also reported this result and said that they felt they were on the verge of solving the general case. (However, at last report, they had not succeeded.) In 1968, John McKay reported from the Atlas Computer Laboratory in England that with the aid of two colleagues, J. Leech and M. S. Patterson, and a computer that he had shown that Klein's 168 group is a factor group of the special group. The homomorphisms differed in detail, but a typical one would send $a$ to $(12)(3456)(7)$ and $c$ to $(1736)(25)(4)$. In 1968 J. M. Van Buskirk reported that $\pi_{1}\left(C^{3}\left(T_{2}\right) \cup P^{3}\right)$ is nontrivial for all positive values of $n$ if and only if it is nontrivial for all negative values.

THEOREM 2.1. For each integer $n \neq 0$, no homotopy 3-cell results from sewing $a$ pillbox onto a cube with a figure eight knotted hole along an annulus that runs through the hole $n$ times.

Proof. For $n \neq 0$, we show the nontriviality of the group

$$
\left\{a, c: a c a^{-1} c^{-1} a c^{-1} a^{-1} c a c^{-1}=\left(c a^{-1} c^{-1} a^{2} c^{-1} a^{-1} c\right)^{n} a=1\right\} .
$$

First we simplify the presentation. If we let $w=c a^{-1}$ and eliminate $c$ by replacing it with $w a$, the first relation is shortened to $a w a^{-1} w^{-2} a^{-1} w a w^{-1}=1$. If we let $k=w^{-1} a$ and replace $a$ by $w k$, the relation becomes $w k^{2} w=k w^{3} k$. The presentation of the group is changed to

$$
\left\{w, k: k w^{3} k=w k^{2} w,\left(w k^{-1} w^{-1} k w^{-1} k^{-1} w k\right)^{n}(w k)=1\right\} .
$$

Now we simplify the second relation. The first relation $w k^{2} w=k w^{3} k$ implies that the factors $w k^{-1} w^{-1} k w^{-1} k^{-1} w k$ and $w k$ commute. (This is also implied by the fact that $w k^{-1} w^{-1} k w^{-1} k^{-1} w k$ and $w k$ are represented by curves on the boundary of a tubular neighborhood of $T_{2}$ and the fundamental group of a 2-dimensional torus is abelian.) Hence the second relations becomes $\left(k^{-2} w^{-1} k w^{-1} k^{-2} w^{-1}\right)^{n}(w k)^{3 n+1}=1$. The first relation simplifies this so we obtain

$$
\pi_{1}\left(C^{3}\left(T_{2}\right) \cup P^{3}\right)=\left\{w, k ; k w^{3} k=w k^{2} w,\left(k w^{4} k^{2}\right)^{n}=(w k)^{3 n+1}\right\} .
$$

We complete the proof of Theorem 2.1 by considering two cases.

Case 1. $n=-1$. In this case the presentation reduces to

$$
\left\{x, y: x y^{2} x=y x^{3} y, y x^{4} y=x y x\right\} .
$$

The substitution $z=x y^{-1}$ changes it to $\left\{x, z ; x^{4} z^{-1} x^{-1} z x^{-1}=z x z^{-1}=x^{-1} z\right.$, which 
simplifies to $\left\{x, z ; x^{7}=z^{3}=(x z)^{2}\right\}$. This group is nontrivial since there is a homomorphism of it onto the group $G=\left\{u, v ; u^{7}=v^{3}=(u v)^{2}=1\right\}$. It was noted on p. 35 of [2] that $G$ is nontrivial since there is a homomorphism of $G$ onto the permutation group generated by $u=(1234567), v=(721)(643)(5)$.

Case 2. $n \neq 0,-1$. In this case $|3 n+1|>2$. By adding the relations $w^{4}=1, k^{3}=1$, we find that there is a homomorphism of $\pi_{1}\left(C^{3}\left(T_{2}\right) \cup P^{3}\right)$ onto $\left\{x, y: x^{4}=y^{3}\right.$ $\left.=\left(y x^{-1}\right)^{3}=(x y)^{3 n+1}=1\right\}$. We show in $\S 4$ that this group is nontrivial if $|3 n+1|>2$.

THEOREM 2.2. The figure eight knot has Property $\mathrm{P}$.

Proof. Theorem 2.1 shows that $T_{2}$ satisfies the first half of Property P. To see that the second condition is satisfied, regard $C^{3}\left(T_{2}\right)$ as standardly embedded in $E^{3}$. Let $h: C^{3}\left(T_{2}\right) \rightarrow E^{3}$ be a piecewise linear homeomorphism. Let $J_{1}$ be a polygonal simple closed curve on $\mathrm{Bd} C^{3}\left(T_{2}\right)$ that goes around, but not through the hole. Since the closure (in $\left.S^{3}\right)$ of one complementary domain of $h\left(\mathrm{Bd}\left(C^{3}\left(T_{2}\right)\right)\right.$ ) is a solid torus, there is a polygonal simple closed curve $J_{2}$ on $\mathrm{Bd}\left(C^{3}\left(T_{2}\right)\right)$ such that $h\left(J_{2}\right)$ bounds a disk $D^{2}$ in $h\left(J_{2}\right) \cup\left(E^{3}-h\left(C^{3}\left(T_{2}\right)\right)\right)$. Notice that $\pi_{1}\left(D^{2} \cup h\left(C^{2}\left(T_{2}\right)\right)\right)$ is trivial.

Now if $J_{1}$ is isotopic to $J_{2}$ on $\mathrm{Bd}\left(C^{3}\left(T_{2}\right)\right)$ then there is a polyhedral 3-cell $P^{3}$ in $\mathrm{Cl}\left(E^{3}-C^{3}\left(T_{2}\right)\right)$ whose intersection with $C^{3}\left(T_{2}\right)$ is an annulus having $J_{2}$ for a centerline. Then $h$ can be extended to take $P^{3}$ onto a regular neighborhood of $D^{2}$ and then to take $E^{3}$ onto $E^{3}$.

If $J_{2}$ is not isotopic to $J_{1}$ on $\mathrm{Bd}\left(C^{3}\left(T_{2}\right)\right)$, it runs through the hole. Since $\pi_{1}\left(D^{2} \cup h\left(C^{3}\left(T_{2}\right)\right)\right)$ is trivial, a homotopy 3-cell results from sewing a pillbox onto $C^{3}\left(T_{1}\right)$ along an annulus whose centerline is $J_{2}$. This violates Theorem 2.1.

3. Cubes with twist knotted holes. A twist knot is a simple closed curve in $E^{3}$ which is doubled [23], with twists about an unknotted circle. See Figures 2 and 3. Twist knots are alternating knots and are determined by the number of half twists. This class includes the trefoil knot ( 1 half twist), the figure eight ( 2 half twists) and the stevedore's knot (4 half twists). In this section we show that cubes with twist knotted holes have Property P. We let $T_{j}$ denote a twist knot with $j$ half twists.

Figure 2 shows a twist knot $T_{2 m}$ with an even number of half twists. In computing $\pi_{1}\left(E^{3}-T_{2 m}\right)$ we use $a_{1}, a_{2}, \ldots, a_{m+1}, b_{1}, b_{2}, \ldots, b_{m+1}$ as generators. Since all the generators are functions of $a_{1}$ and $b_{1}$ we drop their subscripts in writing the relation as follows:

$$
\begin{aligned}
b_{2}= & a_{1}^{-1} b_{1} a_{1}=a^{-1} b a \\
a_{2}= & b_{2} a_{1} b_{2}^{-1}=\left(a^{-1} b\right) a\left(a^{-1} b\right)^{-1} \\
b_{3}= & a_{2}^{-1} b_{2} a_{2}=\left(a^{-1} b\right)^{2} b\left(a^{-1} b\right)^{-2} \\
& \vdots \\
b_{m+1}= & a_{m}^{-1} b_{m} a_{m}=\left(a^{-1} b\right)^{m} b\left(a^{-1} b\right)^{-m} \\
a_{m+1}= & b_{m+1} a_{m} b_{m+1}^{-1}=\left(a^{-1} b\right)^{m} a\left(a^{-1} b\right)^{-m} \\
b_{1}=b= & a_{m+1} a_{1} a_{m+1}^{-1}=\left(a^{-1} b\right)^{m} a\left(a^{-1} b\right)^{-m} a\left(a^{-1} b\right)^{m} a^{-1}\left(a^{-1} b\right)^{-m} .
\end{aligned}
$$




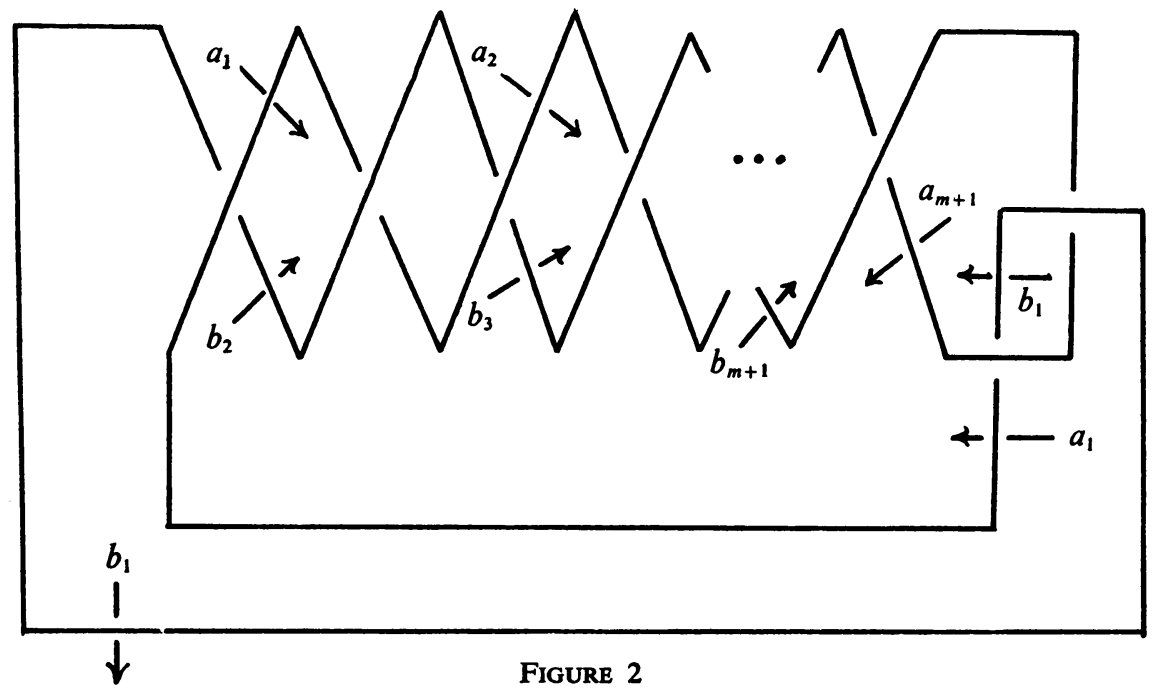

There is another relation given by the rightmost crossing but we ignore it since any one relation is a consequence of the others.

Letting $w=a^{-1} b$ and replacing $b$ with $a w$ we find that

$$
\pi_{1}\left(E^{3}-T_{2 m}\right)=\left\{a, w: a w=w^{m} a w^{-m} a w^{m} a^{-1} w^{-m}\right\} .
$$

Substituting $k=a w^{-m}$ and replacing $a$ with $k w^{m}$ leads to the further simplification

$$
\pi_{1}\left(E^{3}-T_{2 m}\right)=\left\{w, k ; k w^{2 m+1} k=w^{m} k^{2} w^{m}\right\} .
$$

Figure 3 shows a twist knot $T_{2 m-1}$ with an odd number of half twists. Again, letting $a_{1}=a$ and $b_{1}=b$ we find that the crucial relation is

$$
b=b_{m+1}^{-1} a b_{m+1}=\left(a^{-1} b\right)^{m} b^{-1}\left(a^{-1} b\right)^{-m} a\left(a^{-1} b\right)^{m} b\left(a^{-1} b\right)^{-m} \text {. }
$$

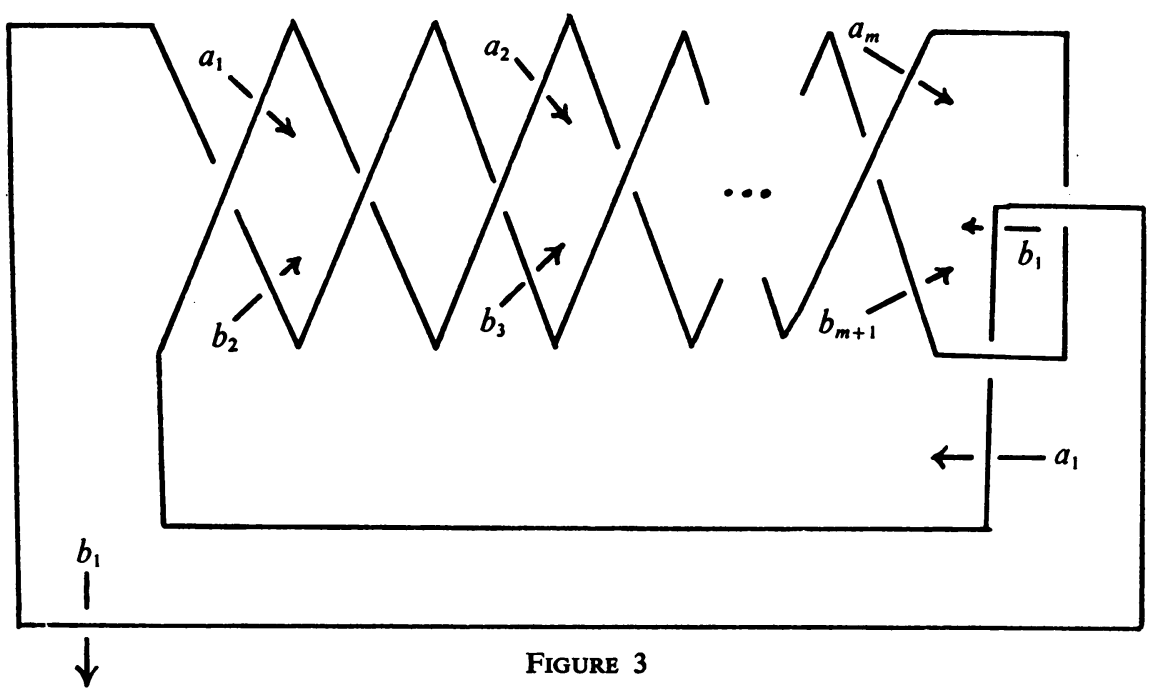


By letting $w=a^{-1} b$ and eliminating $a$ we obtain $b=w^{m} b^{-1} w^{-m} b w^{-1} w^{m} b w^{-m}$. Substituting $k=b w^{-m}$ and eliminating $b$ we obtain

$$
\pi_{1}\left(E^{3}-T_{2 m-1}\right)=\left\{w, k: k w^{2 m-1} k=w^{m} k^{2} w^{m}\right\} .
$$

In the last section we converted a cube with a figure eight knotted hole into a homology 3-cell by sewing on a pillbox along an annulus that runs through the hole $n$ times. If we do the same with $C^{3}\left(T_{2 m}\right)$ the added relation is

$$
\left(a_{m+1}^{-1} a_{1} a_{2} \cdots a_{m} b_{1}^{-1} b_{m+1} \cdots b_{2} a^{2-2 m}\right)^{n} a=1
$$

where $a=a_{1}$, and $a$ and the expression in the parentheses commute. Introducing $w=a^{-1} b$ to eliminate $b$ we obtain

$$
\left(w^{m} a^{-1} w^{-m}(a w)^{m} w^{-1-m} a^{-1} w^{m} a^{2-m}\right)^{n} a=1 .
$$

Since $a w=w^{m} a w^{-m} a^{-1} w^{-m}$, then $(a w)^{m}=w^{m} a w^{-m} a^{m} w^{m} a^{-1} w^{-m}$, and we get the simplification $\left(w^{m} a^{-1} w^{-2 m-1} a^{-1} w^{m} a^{2}\right)^{n} a=1$. By letting $k=a w^{-m}$ and using the commuting relation, we find that

$$
\pi_{1}\left(C^{3}\left(T_{2 m}\right) \cup P^{3}\right)=\left\{w, k: k w^{2 m+1} k=w^{m} k^{2} w^{m},\left(k^{2} w^{3 m+1} k\right)^{n}=\left(k w^{m}\right)^{3 n+1}\right\} .
$$

For $\left.\pi_{1}\left(C^{3} T_{2 m-1}\right) \cup P^{3}\right)$, the extra relation is

$$
\left(a_{1} a_{2} \cdots a_{m} b_{1} b_{m} b_{m-1} \cdots b_{2} b_{m+1} b_{1}^{-1-2 m}\right)^{n} b=1 .
$$

A simplification after letting $w=a^{-1} b$ yields $\left(w^{-m} b w^{2 m-1} b w^{-m} b^{-2}\right)^{n} b=1$, and the substitution $k=b w^{-m}$ shows that

$$
\pi_{1}\left(C^{3}\left(T_{2 m-1}\right) \cup P^{3}\right)=\left\{w, k ; k w^{2 m-1} k=w^{m} k^{2} w^{m},\left(k^{2} w^{3 m-1} k\right)^{n}=\left(k w^{m}\right)^{3 n-1}\right\} .
$$

TheOREM 3.1. The twist knots $T_{j}, j \neq 0$, have Property P.

Proof. In order to establish Theorem 3.1, it is sufficient to show that $\pi_{1}\left(C^{3}\left(T_{j}\right) \cup P^{3}\right)$ is nontrivial unless $n=0$.

We first consider the case where $j=2 m$. In this case we are interested in the group with generators $w, k$ and relations (1) $k w^{2 m+1} k=w^{m} k^{2} w^{m}$ and (2) $\left(k^{2} w^{3 m+1} k\right)^{n}$ $=\left(k w^{m}\right)^{3 n+1}$. Now if we adjoin the relations (3) $w^{3 m+1}=1$ and (4) $k^{3}=1$, then (1) becomes (1) $\left(w^{m} k^{-1}\right)^{3}=1$ and (2) becomes (2) $\left(k w^{m}\right)^{3 n+1}=1$. Now since $w^{3 m+1}=1$ this group is generated by $w^{m}$ and $k$ and hence is isomorphic with

$$
\left\{a, b: a^{3 m+1}=b^{3}=\left(a b^{-1}\right)^{3}=(a b)^{3 n+1}=1\right\} .
$$

In the next section we show that this group is nontrivial if $|3 n+1|>2$.

We make a special argument for the case $n=-1$. In this case we do not adjoin the relations $w^{3 m+1}=1=k^{3}$ but instead consider the group generated by $w$ and $k$ with relations (1) $k w^{2 m+1} k=w^{m} k^{2} w^{m}$ and (2) $\left(k^{2} w^{3 m+1} k\right)^{-1}=\left(k w^{m}\right)^{-2}$, or (2) $k w^{3 m+1} k=w^{m} k w^{m}$. We may write out (1) as $\left(w^{m} k w^{m}\right) w^{-2 m}\left(w^{m} k w^{m}\right)=k w^{2 m+1} k$ and then substitute (2) in (1) arriving at (1) $k^{-1} w^{2 m} k^{-1}=w^{4 m+1}$. Making the substitution 
$k=w^{m} x^{-1}$ we have (1) $x w^{m} x w^{m}=w^{6 m+1}$, and (2) $w^{6 m+1}=w^{m} x w^{m} x^{-1} w^{m} x w^{m}$. Substituting (1) in (2), we have

(1) $w^{6 m+1}=\left(x w^{m}\right)^{2}$ and

(2) $\left(x w^{m}\right)^{2}=x^{3}$, and so our group is $\left\{x, w ; w^{6 m+1}=x^{3}=\left(x w^{m}\right)^{2}\right\}$.

There is a homomorphism of this group onto $\left\{x, w ; w^{6 m+1}=x^{3}=\left(x w^{m}\right)^{2}=1\right\}$ and since this group is generated by $x$ and $w^{m}$ it is isomorphic with $\left\{a, b ; a^{6 m+1}=b^{3}\right.$ $\left.=(a b)^{2}=1\right\}$. In [2], it is shown that this group is nontrivial, since there is a homomorphism of it onto the permutation group generated by $a=(1,2, \ldots, 6 m+1)$ and $b=(6 m+1,2,1)(6 m, 4,3) \cdots(4 m+2,4 m, 4 m-1)(4 m+1)$.

We now turn our attention to the case where $j=2 m-1$. In this case we are interested in the group with generators $w, k$, and relations (1) $k w^{2 m-1} k=w^{m} k^{2} w^{m}$ and (2) $\left(k^{2} w^{3 m-1} k\right)^{n}=\left(k w^{m}\right)^{3 n-1}$. If we adjoin the relations (3) $w^{3 m-1}=1$ and (4) $k^{3}=1$ then we have (1) $\left(k w^{-m}\right)^{3}=1$ and (2) $\left(k w^{m}\right)^{3 n-1}=1$. Now since $w^{3 m-1}=1$ this group is generated by $w^{m}$ and $k$ and is isomorphic with $\left\{a, b ; a^{3 m-1}=b^{3}=\left(a b^{-1}\right)^{3}\right.$ $\left.=(a b)^{3 n-1}=1\right\}$. In the next section we show that this group is nontrivial unless $3 m-1=2$ or $3 n-1=2$. In case $m=1$, the knot under consideration is the trefoil, which is known to have Property $\mathrm{P}$ [2]. We handle the case $n=1$ by a special method. We do not add the relations $w^{3 m-1}=1=k^{3}$ but consider the group generated by $w$ and $k$ with relations (1) $k w^{2 m-1} k=w^{m} k^{2} w^{m}$ and (2) $k^{2} w^{3 m-1} k$ $=\left(k w^{m}\right)^{2}$ or (2) $k w^{3 m-1} k=w^{m} k w^{m}$. We rewrite (1) as $\left(w^{m} k w^{m}\right) w^{-2 m}\left(w^{m} k w^{m}\right)$ $=k w^{2 m-1} k$ and then substitute (2) in (1) to obtain

(1) $k w^{-2 m} k=w^{1-4 m}$ and

(2) $k w^{3 m-1} k=w^{m} k w^{m}$.

We now make the substitution $k=x w^{m}$ and arrive at (1) $x w^{-m} x w^{-m}=w^{1-6 m}$ and (2) $w^{1-6 m}=w^{-m} x w^{-m} x^{-1} w^{-m} x w^{-m}$. Now substituting (1) in (2) we have (1) $w^{1-6 m}=\left(x w^{-m}\right)^{2}$ and (2) $x^{3}=\left(x w^{-m}\right)^{2}$. This gives the group $\left\{x, w ; w^{1-6 m}=x^{3}\right.$ $\left.=\left(x w^{-m}\right)^{2}\right\}$. Now there is a homomorphism of this group onto $\left\{x, w ; w^{1-6 m}=x^{3}\right.$ $\left.=\left(x w^{-m}\right)^{2}=1\right\}$ which in turn is isomorphic with $\left\{a, b ; a^{6 m-1}=b^{3}=(a b)^{2}=1\right\}$. In [2] it is shown that this group is nontrivial, since there is a homomorphism of it onto the permutation group generated by

$$
a=(1,2, \ldots, 6 m-1)
$$

and

$$
b=(6 m-1,2,1)(6 m-2,4,3) \cdots(4 m+1,4 m-1,4 m-3)(4 m)(4 m-2) .
$$

This concludes the proof of Theorem 3.1.

4. The nontriviality of certain groups. The groups $(l, p \mid q, r)=\left\{a, b ; a^{l}=b^{p}\right.$ $\left.=(a b)^{q}=\left(a^{-1} b\right)^{r}=1\right\}$ have been studied by Coxeter [8], [9] and others. In particular, in [9] $\mathrm{H}$. Coxeter establishes finiteness theorems for $(3, p \mid q, r)$ and shows that $(3, p \mid 3, r)$ is nontrivial if $p, r \geqq 3$. For completeness we include a proof of the nontriviality of the groups $\left\{a, b: a^{3}=b^{p}=(a b)^{3}=\left(a^{-1} b\right)^{r}\right\}$ if $p, r \geqq 3$. We first notice that this group is isomorphic with $\left\{A, B: A^{3}=B^{3}=(A B)^{r}=\left(A^{-1} B\right)^{p}\right.$ 
$=1\}$ by sending $a$ to $A$ and $a b$ to $B$. This group in turn is isomorphic with $\left\{C, D: C^{3}=D^{3}=(C D)^{p}=\left(C^{-1} D\right)^{r}=1\right\}$ and hence in $\left\{A, B: A^{3}=B^{3}=(A B)^{r}\right.$ $\left.=\left(A^{-1} B\right)^{p}=1\right\}$ we may assume that $r \geqq p \geqq 3$.

We now choose a complex number $c$ such that $c \bar{c}=4 \cos ^{2}(\pi / r)$ and $c+\bar{c}$ $=4 \cos ^{2}(\pi / p)-4 \cos ^{2}(\pi / r)-1$. This choice is always possible if $r \geqq p \geqq 3$. Let $A$ be the $3 \times 3$ matrix

$$
\left[\begin{array}{rrr}
1 & c & c+1 \\
0 & -1 & -1 \\
0 & 1 & 0
\end{array}\right]
$$

and $B$ be the matrix

$$
\left[\begin{array}{rrr}
0 & 0 & 1 \\
1+\bar{c} & 1 & 1 \\
-1 & 0 & -1
\end{array}\right]
$$

Then

$$
A B=\left[\begin{array}{rrr}
c \bar{c}-1 & c & 0 \\
-\bar{c} & -1 & 0 \\
1+\bar{c} & 1 & 1
\end{array}\right] \text { and } A^{-1} B=\left[\begin{array}{rrr}
c+\bar{c}+c \bar{c} & c+1 & c+1 \\
-1 & 0 & -1 \\
-\bar{c} & -1 & 0
\end{array}\right]
$$

Now computing characteristic polynomials we have

$$
\begin{gathered}
P_{A}(x)=1-x^{3}, \quad P_{B}(x)=1-x^{3}, \\
P_{A B}(x)=1-(c \bar{c}-1) x+(c \bar{c}-1) x^{2}-x^{3}=-(x-1)\left(x^{2}-2 \cos (2 \pi / r) x+1\right),
\end{gathered}
$$

and

$P_{A^{-1} B}(x)=1-(c+\bar{c}+c \bar{c}) x+(c+\bar{c}+c \bar{c}) x^{2}-x^{3}=-(x-1)\left(x^{2}-2 \cos (2 \pi / p) x+1\right)$.

Now $P_{A B}$ divides $x^{r}-1$ and $P_{A^{-1} B}$ divides $x^{p}-1$.

Since a matrix satisfies its characteristic polynomial [6, p. 299], this shows that $A$ and $B$ satisfy the relations $A^{3}=B^{3}=(A B)^{r}=\left(A^{-1} B\right)^{p}=1$.

5. Other knots. In this section we will show that many other knots have Property P. First, we need some additional definitions.

Let $M$ be a homotopy 3-sphere and let $S$ be a polyhedral subset of $M$ which is homeomorphic with $S^{1} \times S^{1}$. Then it follows from the loop theorem and Dehn's lemma [17] that there is a disk $D$ such that $D \cap S=\mathrm{Bd} D$ and $\mathrm{Bd} B$ is not homotopic to 0 on $S$. The closure of the complementary domain of $S$ containing Int $D$ is then a homotopy solid torus, that is, homeomorphic with a homotopy 3-cell with a pair of disks on its boundary identified.

If $K_{1}$ and $K_{2}$ are knots in $S^{3}$, then the sum of $K_{1}$ and $K_{2}$, denoted $K_{1}+K_{2}$ is described in [10]. For example, the square knot is a composite. 
Suppose that $M$ is a 3-manifold, and $T$ is a solid torus in Int $M$. If we remove Int $T$ from $M$ and then sew back a solid torus $T^{\prime}$ so that a meridianal curve of $T^{\prime}$ is not isotopic on $\mathrm{Bd} T$ with a meridianal curve of $T$, then we obtain a 3-manifold $M^{\prime}$. In this case we will say that $M^{\prime}$ is obtained from $M$ by removing $T$ and sewing it back differently.

\section{THEOREM 5.1. If $K$ is a composite knot, then $K$ has Property $\mathrm{P}$.}

Theorem 5.1 is only a slight extension of the result by D. Noga [16, Satz 1], that one cannot recover $S^{3}$ by removing a solid torus about $K$ and sewing it back differently. Also Theorem 5.1 is a special case of a more general theorem of Conner [7] in which he shows that "splittable" knots have Property P.

Before proceeding with the proof of Theorem 5.1, we first prove two lemmas.

LEMma 1. Suppose that $T$ is a homotopy solid torus, and $K$ is a cube with a knotted hole in the interior of $T$. Then there is a homotopy 3-cell $B$ in $T$ such that $K \subset B$.

Proof. Let $D$ be a disk in $T$ such that $D \cap \operatorname{Bd} T=\operatorname{Bd} D$, and $\mathrm{Bd} D$ is not homotopic to 0 in $\mathrm{Bd} T$. Putting $D$ in general position with respect to $\mathrm{Bd} K$ we find that $D \cap \mathrm{Bd} K$ is a finite collection of mutually disjoint simple closed curves. We may assume that each curve of intersection is not homotopic to 0 on $\mathrm{Bd} K$, for these may be removed by a simple disk replacement. Let $J$ be an innermost curve of intersection on $D$ and let $H$ be the subdisk of $D$ bounded by $J$. It is impossible that $H \subset K$, since $K$ is a cube with a knotted hole and $i_{*}: \pi_{1}(\mathrm{Bd} K) \rightarrow \pi_{1}(K)$ is one-toone. By adding a regular neighborhood of $H$ to $K$ we obtain a homotopy 3-cell which contains $K$. If there are no curves of intersection then the removal of the interior of a regular neighborhood of $D$ from $T$ results in a homotopy 3-cell which contains $K$. This establishes Lemma 1 .

Lemma 2. Let $T_{1}$ be a solid torus and let $T_{2}$ be a solid torus in the interior of $T_{1}$ such that (a) there is a meridianal disk of $T_{1}$ whose intersection with $T_{2}$ is a meridianal disk of $T_{2}$, and (b) $T_{2}$ is not parallel to $T_{1}$. See Figure 4. Then the result of removing $T_{2}$ from $T_{1}$ and sewing it back differently is not a homotopy solid torus.

Proof. First, let $F$ be a meridianal disk of $T_{1}$ which intersects $T_{2}$ in a meridianal disk of $T_{2}$. Let $N$ be a regular neighborhood in $T_{1}$ of $F$ and let $K_{1}$ be the closure of $T_{1}-\left(N \cup T_{2}\right)$. Then $K_{1}$ is a cube with a knotted hole since $T_{2}$ is not parallel to $T_{1}$. Now $K_{1} \cap \mathrm{Bd} T_{1}$ is an annulus and we push this annulus slightly to the interior of $T_{1}$ and continue to call the resulting cube with a knotted hole $K_{1}$. Suppose that we remove $T_{2}$ from $T_{1}$ and sew it back differently. Call the resulting manifold $T_{1}^{\prime}$, and the replaced $T_{2}, T_{2}^{\prime}$. Suppose that $T_{1}^{\prime}$ is a homotopy solid torus. Then there is a disk $D$ in $T_{1}^{\prime}$ such that $D \cap \operatorname{Bd} T_{1}^{\prime}=\mathrm{Bd} D$ and $\mathrm{Bd} D$ is not homotopic to 0 on $\mathrm{Bd} T_{1}^{\prime}$. Now since, by Lemma $1, K_{1}$ lies in a homotopy 3-cell in $T_{1}^{\prime}$, we may assume that $D \cap K_{1}=\varnothing$. We now put $D$ and $\mathrm{Bd} T_{2}$ in general position so that $D \cap \operatorname{Bd} T_{2}$ is a 


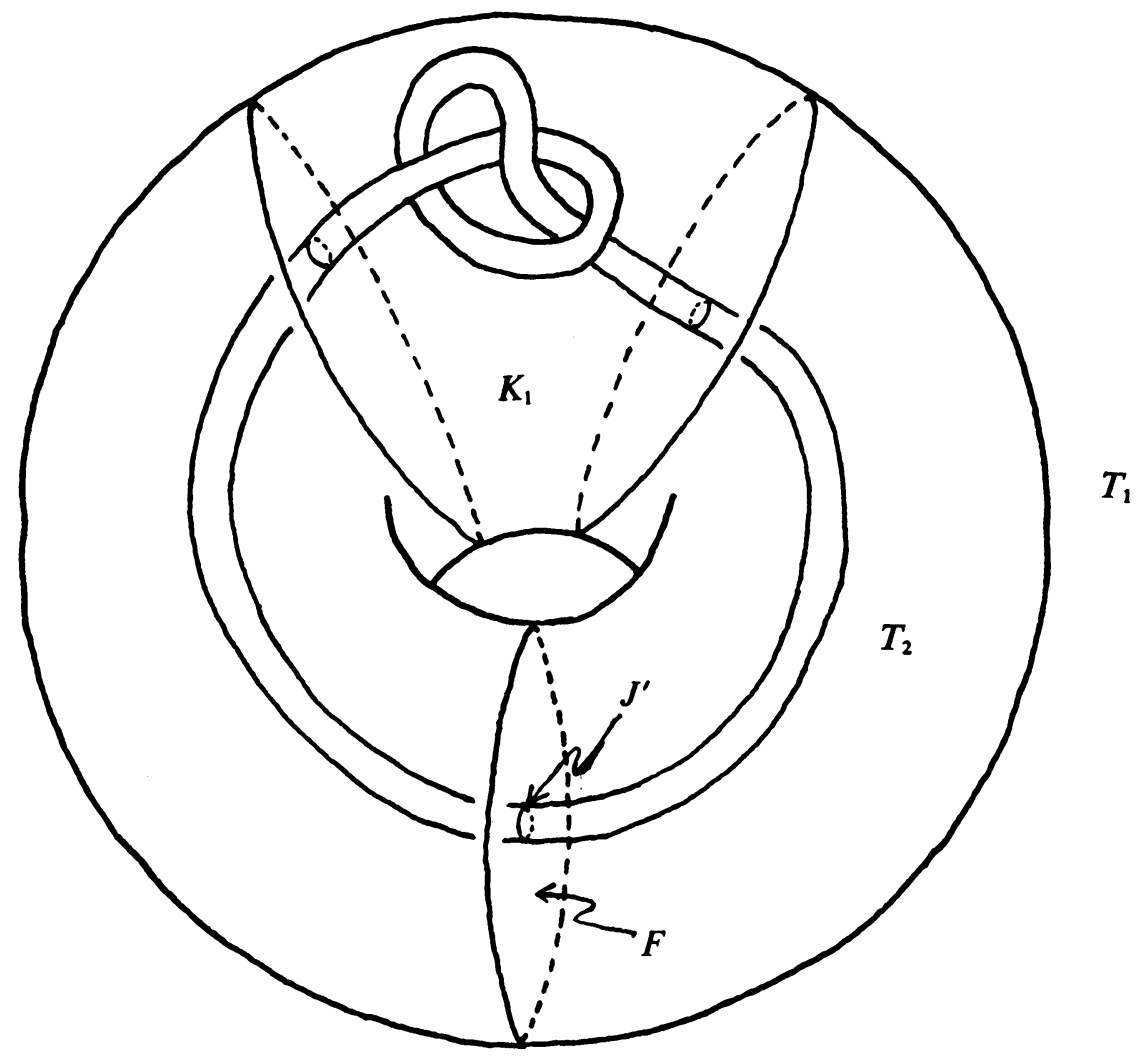

FIGURE 4

finite collection of mutually disjoint simple closed curves. We may assume that none of these simple closed curves is homotopic to 0 on $\mathrm{Bd} T_{2}$. Since $D \cap K_{1}=\varnothing$, it follows that each curve of intersection of $D \cap \mathrm{Bd} T_{2}$ is homotopic to the simple closed curve $J^{\prime}=F \cap \mathrm{Bd} T_{2}$ that was originally meridianal on $T_{2}$. Let $J$ be an innermost curve of intersection on $D$ and let $H$ be the subdisk of $D$ bounded by $J$. Now $H$ is not contained in $T_{2}^{\prime}$ for otherwise $T_{2}$ would not have been sewn in differently. But $H$ is not contained in $\mathrm{Cl}\left(T_{1}^{\prime}-T_{2}^{\prime}\right)$ since $J^{\prime}$ is not homotopic to 0 in $\mathrm{Cl}\left(T_{1}^{\prime}-T_{2}^{\prime}\right)$. Consequently there are no curves of intersection. But then $\mathrm{Bd} D$ is homotopic to 0 in $\mathrm{Cl}\left(T_{1}^{\prime}-T_{2}^{\prime}\right)$ and hence in $\mathrm{Bd} T_{1}^{\prime}$. This contradicts the choice of $D$. Hence $T_{1}^{\prime}$ is not a homotopy solid torus. This establishes Lemma 2.

Proof of Theorem 5.1. Let $K=K_{1}+K_{2}$ be a composite knot in $S^{3}$. We will show that the result of removing a regular neighborhood of $K$ and sewing it back differently is not a simply connected manifold. It is easy to see that this process is equivalent to attaching a pillbox to a cube with a $K$-knotted hole in a nonstandard way. Let $S$ by a polyhedrally embedded $S^{1} \times S^{1}$ which envelopes the knot $K_{1}$ and follows the knot $K_{2}$. See Figure 5 . Notice that $\left(S^{3}\right.$-interior $\left.S\right)$ is a cube with a knotted hole and hence is not a homotopy solid torus. 
Now let $N$ be a regular neighborhood of $K$ in interior $S$ and let $M$ be a 3-manifold which results from removing $N$ from $S^{3}$ and sewing it back differently. Now it follows from Lemma 2 that the torus $S$ in $M$ does not bound a homotopy solid torus in $M$. Hence $M$ is not simply connected. This establishes Theorem 5.1.

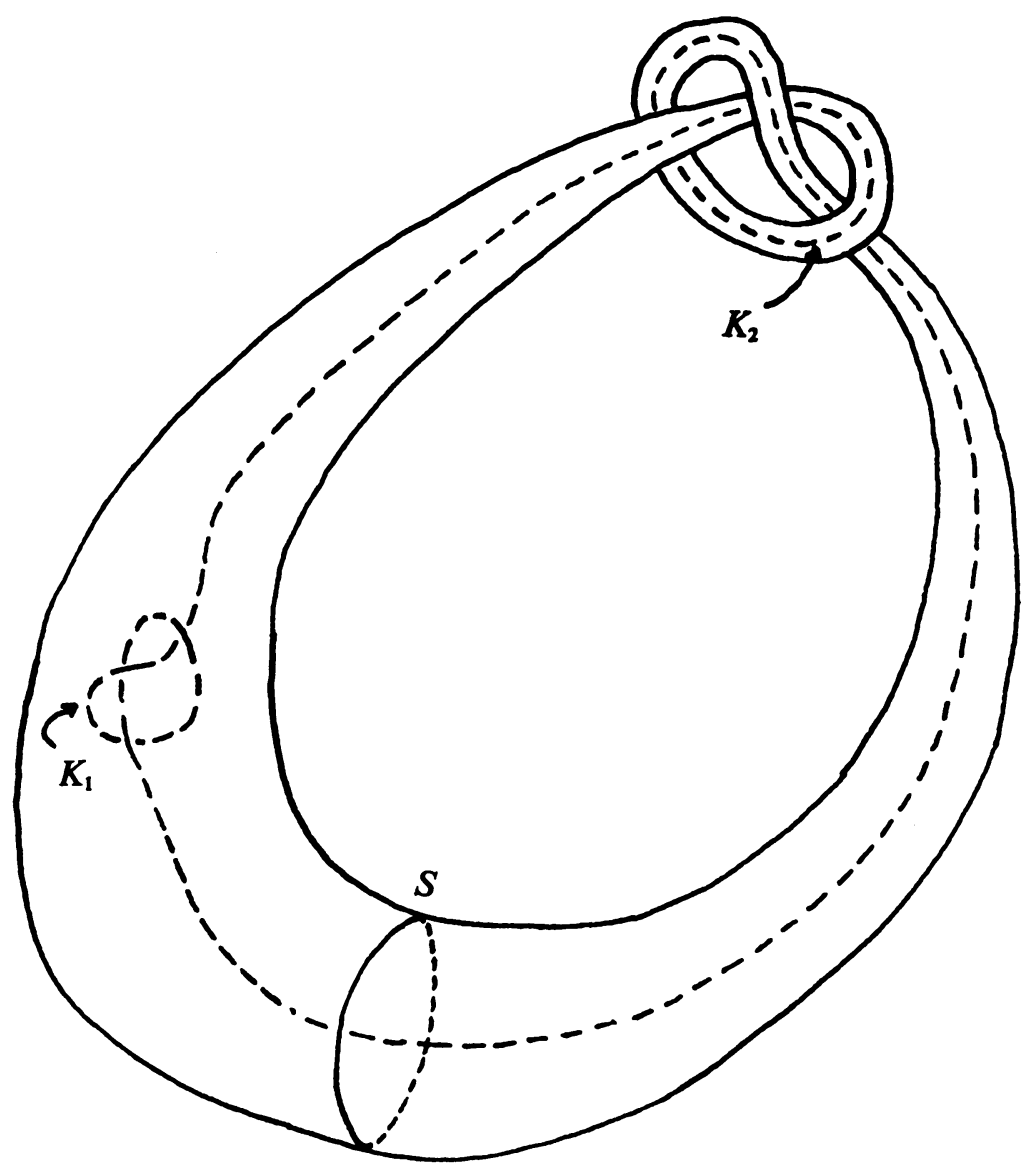

FIGURE 5

Theorem 5.2. Suppose that $K$ is a knot in $S^{3}$ with Property $\mathrm{P}$ and that $T$ is an unknotted polyhedral solid torus in $S^{3}$ which contains $K$ in its interior. Let $L$ be a simple closed curve on $\mathrm{Bd} T$ which bounds a disk in $S^{3}-\mathrm{Int} T$ but not on $\mathrm{Bd} T$, and let $h: T \rightarrow S^{3}$ be a piecewise linear homeomorphism such that $h(L)$ is homologous to 0 in $S^{3}-$ Int $[h(T)]$. Then $h(K)$ has a Property $\mathrm{P}$.

Proof. Let $N$ be a regular neighborhood of $K$ in Int $T$ and let $M$ be a meridianal simple closed curve on Bd $T$ which is transverse to $L$.

Suppose that we remove $N$ from $T$ and sew it back differently, arriving at the manifold $T^{\prime}$. Since $K$ has Property $\mathrm{P}$, there are two possibilities. First, $T^{\prime}$ may not 
be a homotopy solid torus, or, secondly, $T^{\prime}$ is a homotopy solid torus whose meridianal curve is $p M+q L$ where $p$ and $q$ are relatively prime integers and $|p| \neq 1$.

Now, suppose that we remove $h(N)$ from $S^{3}$ and sew it back differently, obtaining the manifold $M^{3}$. If $h(T)$ is unknotted, then $h$ may be extended to a homeomorphism of $S^{3}$ onto $S^{3}$. Since $K$ has Property $\mathrm{P}$, it follows that in this case $M^{3}$ is not simply connected.

If $h(T)$ is knotted and the result of sewing back $h(N)$ in $h(T)$ is not a homotopy solid torus, then $M^{3}$ is not simply connected since $h(\mathrm{Bd} T)$ does not bound a homotopy solid torus in $M^{3}$.

Finally, suppose that $h(T)$ is knotted and the result of sewing back $h(N)$ in $h(T)$ is a homotopy solid torus. Then a meridianal curve for this homotopy solid torus is $p h(M)+q h(L),|p| \neq 1$, and it follows that the first homology group of $M^{3}$ is either $Z$ or $Z_{p}$. In any case $M^{3}$ is not simply connected. This establishes Theorem 5.2.

\section{Corollary 5.2. Doubled knots [21] with $\rho$ twists $\rho \neq 0$ have Property P.}

Proof. This follows directly from the definition of doubled knots, Theorem 3.1 and Theorem 5.2. Actually, it is true that doubled knots with no twists also have Property $\mathbf{P}$ if they are knotted. We will show this in a later paper.

6. Questions. In this section we raise some questions related to Property $P$.

(1) In examining the knot table of Alexander and Briggs [1], one finds that the first two knots which are not known to have Property $P$ are $6_{2}$ and $6_{3}$. These knots are shown in Figures 6 and 7, respectively. Do these knots have Property P?

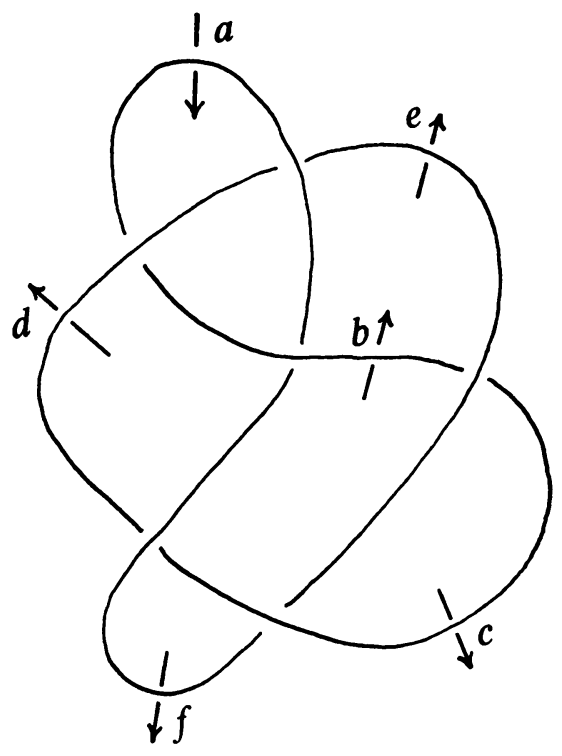

FIGURE 6

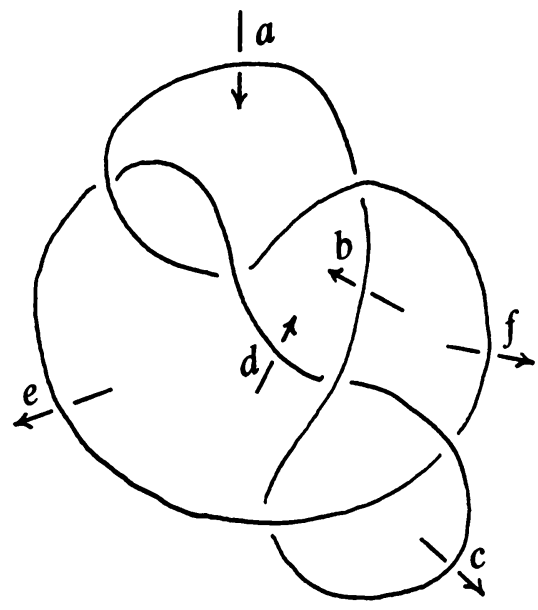

FIGURE 7 
If one converts the cube with a knotted hole $C^{3}\left(6_{2}\right)$ to a homology cube by sewing on a pillbox $P^{3}$, along an annulus which runs through the hole $|n|$ times, one presentation of $\pi_{1}\left(C^{3}\left(6_{2}\right) \cup P^{3}\right)$ has generators $a, b, c, d, e$ and $f$ and relations $b=d a d^{-1}, c=e^{-1} b e, d=f^{-1} c f, e=a d a^{-1}, f=c^{-1} e c$, and $\left(b^{-1} c^{-1} a f^{-1} e^{-1} d a^{2}\right)^{h} a=1$.

If one converts the cube with a knotted hole $C^{3}\left(6_{3}\right)$ to a homology cube by sewing on a pillbox $P^{3}$, along an annulus which runs through the hole $|n|$ times, one presentation of $\pi_{1}\left(C^{3}\left(6_{3}\right) \cup P^{3}\right)$ has generators $a, b, c, d, e$ and $f$, and relations $b=f a f^{-1}, c=e^{-1} b e, d=b^{-1} c b, e=a d a^{-1}, f=c^{-1} e c$, and $\left(f^{-1} e b a^{-1} c d^{-1}\right)^{n} a=1$.

(2) Do knots whose groups have finitely generated commutator subgroups [15] have Property $P$ ?

(3) Do pretzel knots [21] have Property P ?

(4) If the Poincaré conjecture is false, can a homotopy 3-cell which is not a topological 3-cell be obtained by attaching a pillbox to a cube with a knotted hole?

(5) If $k_{1}$ and $k_{2}$ are polygonal knots in $E^{3}$ with homeomorphic complements, is there a homeomorphism of $E^{3}$ onto itself which carries $k_{1}$ onto $k_{2}$ ?

(6) In showing that the figure eight knot (and other twist knots) have Property $P$, we used the fact that certain matrix groups are nontrivial. In dealing with the trefoil (and other torus knots), finite permutation groups sufficed for the same purpose. Is there a collection of nontrivial finite permutation groups such that for each positive integer $n$, each of the groups

$$
\left\{w, k: k w^{3} k=w k^{2} w,\left(k w^{4} k^{2}\right)^{n}=(w k)^{3 n \pm 1}\right\}
$$

maps homomorphically onto one of them? In Case 1 of the proof of Theorem 2.1, we noted that, if $3 n \pm 1=2$, the group maps onto the permutation group generated by $(1,2,3,4,5,6,7)$ and $(7,2,1)(6,4,3)(5)$. Such permutation groups can also be found if $3 n \pm 1$ is divisible by 4,5 or 7 .

(7) Is there a geometric proof that the trefoil knot has Property P? Such an argument might provide a clue for showing that all nontrivial knots have Property P.

\section{REFERENCES}

1. J. W. Alexander and G. B. Briggs, On types of knotted curves, Ann. of Math. 28 (1926), 562-586.

2. R. H. Bing, Necessary and sufficient conditions that a 3-manifold be $S^{3}$, Ann. of Math. (2) 68 (1958), 17-37. MR 20 \#1973.

3. - Correction to "Necessary and sufficient conditions that a 3-manifold be $S^{3}$ ", Ann. of Math. (2) 77 (1963), 210. MR 25 \#5508.

4. - Some aspects of the topology of 3-manifolds related to the Poincaré conjecture, Lectures on Modern Math., vol. 2, Wiley, New York, 1964, pp. 93-128. MR 30 \#2474.

5. - Computing the fundamental group of the complement of curves, Washington State University, Pullman, Wash., 1965.

6. G. Birkhoff and S. Mac Lane, A survey of modern algebra, 3rd ed., Macmillan, New York, 1965. MR 31 \#2250.

7. A. C. Conner, Splittable knots (preprint). 
8. H. S. M. Coxeter, The abstract groups $G^{m, n . p}$, Trans. Amer. Math. Soc. 45 (1939), 73-150.

9. - The abstract group $G^{3.7 .16}$, Proc. Edinburgh Math. Soc. (2) 13 (1962), 47-61, 189. MR 26 \#190; MR 26 \#6267.

10. R. H. Fox, A quick trip through knot theory, Topology of 3-Manifolds and Related Topics (Proc. Univ. of Georgia Inst., 1961), Prentice-Hall, Englewood Cliffs, N. J., 1962, pp. 120-167. MR 25 \#3522.

11. H. Gluck, Ph.D. Thesis, Princeton University, Princeton, N. J., 1961.

12. J. Hempel, Construction of orientable 3-manifolds, Topology of 3-Manifolds and Related Topics (Proc. Univ. of Geogria Inst., 1961), Prentice-Hall, Englewood Cliffs, N. J., 1962, pp. 207-212. MR 25 \#3538.

13. - A simply connected 3-manifold is $S^{3}$ if it is the sum of a solid torus and the complement of a torus knot, Proc. Amer. Math. Soc. 15 (1964), 154-158. MR 28 \#599.

14. W. B. R. Lickorish, A representation of orientable combinatorial 3-manifolds, Ann. of Math. (2) 76 (1962), 531-540. MR 27 \#1929.

15. L. Neuwirth, The algebraic determination of the genus of knots, Amer. J. Math. 82 (1960), 791-798. MR 22 \#11397.

16. D. Noga, Uber den Aussenraum von Produktknoten und die Bedeutung der Fixgruppen, Math. Z. 101 (1967), 131-141. MR 36 \#2137.

17. C. D. Papakyriakopoulos, On solid tori, Proc. London Math. Soc. (3) 7 (1957), 281-299. MR 19, 441.

18. - On Dehn's lemma and the asphericity of knots, Ann. of Math. (2) 66 (1957), 1-26. MR 19, 761.

19. H. Poincaré, Second complément a l'analysis situs, Proc. London Math. Soc. (2) 32 (1900), 277-308.

20. - Cinquieme complément a l'analysis situs, Rend. Circ. Mat. Palermo 18 (1904), $45-110$.

21. H. F. Trotter, Non-invertible knots exist, Topology 2 (1963), 275-280. MR 28 \#1618; errata MR 30 p. 1203.

22. A. H. Wallace, Modifications and cobounding manifolds, Canad. J. Math. 12 (1960), 503528. MR 23 \#A2887.

23. J. H. C. Whitehead, On doubled knots, J. London Math. Soc. 12 (1937), 63-71.

UNIVERSITY OF WISCONSIN,

MADISON, WISCONSIN 53706 